\title{
An improved Ullmann-Ukita-Buchwald-Li conditions for CuI-catalyzed coupling reaction of 2-pyridones with aryl halides
}

\author{
Po-Shih Wang, ${ }^{\text {a,b }}$ Chih-Kai Liang ${ }^{\mathrm{a}, \mathrm{b}}$ and Man-kit Leung ${ }^{\mathrm{a}, \mathrm{b}, *}$ \\ a Department of Chemistry, National Taiwan University, Taipei 106, Taiwan ROC \\ ${ }^{\mathrm{b}}$ Institute of Polymer Science and Engineering, National Taiwan University, Taipei 106, Taiwan ROC
}

Received 20 October 2004; revised 31 December 2004; accepted 5 January 2005

\begin{abstract}
An effective CuI-trans- $N, N^{\prime}$-dimethylcyclohexane-1,2-diamine (DMCDA)- $\mathrm{K}_{2} \mathrm{CO}_{3}$-catalyzed coupling reaction of 2-pyridones with aryl halides is described. Under our conditions, DMCDA was found to be an effective catalyst that facilitates the coupling reactions even in toluene, a common industrial solvent. In addition, 3-bromopyridine could also be coupled effectively under these conditions, indicating that the catalytic reactivity of this system is high. The reaction could be applied for polymer modification and iterative oligo-pyridone synthesis.

(C) 2005 Elsevier Ltd. All rights reserved.
\end{abstract}

\section{Introduction}

$N$-Substituted pyrid-2-ones have attracted synthetic organic chemists because of their chemical reactivity, photochemical behavior, as well as their biological activities. ${ }^{1,2}$ Direct $\mathrm{C}-\mathrm{N}$ bond formation is one of the most convenient synthetic approaches for $\mathrm{N}$-alkylpyridones. ${ }^{3}$ However, synthetic examples for $N$-arylpyridones are rare. ${ }^{4}$ Traditional copper-catalyzed Ullmann type $\mathrm{C}-\mathrm{N}$ bond formation often requires the use of high temperatures as well as stoichiometric amounts of copper reagents. ${ }^{5}$ Recently, palladium catalyzed $N$-arylations have been extensively explored. ${ }^{6}$ In addition, Ullmann-type coppercatalyzed processes for $\mathrm{C}-\mathrm{N}$ bond formation such as $\mathrm{N}$-arylation of amines, ${ }^{7}$ anilines, ${ }^{8}$ amides, ${ }^{9}$ imidazole, ${ }^{10}$ indoles, ${ }^{9}$ and hydrazines ${ }^{11}$ have been reported. The reactivity of the copper catalyst strongly relies on the ligand system used in the reaction. $N, N^{\prime}$-Dimethylethylenediamines and derivatives are known effective ligands to promote the reactions.

Ukita has recently reported the copper-catalyzed Ullmann $\mathrm{N}$-arylation of 2-hydroxypyridine. ${ }^{4 \text { a }}$ Copper halides, oxides, or copper powder facilitates the reaction. However, high temperatures of $120-150{ }^{\circ} \mathrm{C}$ in DMF are usually required. The reaction is sensitive to steric hindrance. Orthosubstituted aryl halides are ineffective toward the coupling reaction.

Keywords: 2-Pyridones; Copper reagents; C-N coupling; Toluene.

* Corresponding author.; e-mail: mkleung@ntu.edu.tw
$\mathrm{Li}$ has recently reported a modification of the Ukita conditions, using $\mathrm{CuI}-\mathrm{MeNHCH} \mathrm{CH}_{2} \mathrm{NHMe}_{2}-\mathrm{K}_{3} \mathrm{PO}_{4}$ in dioxane to promote the coupling reaction. ${ }^{4 \mathrm{~b}}$ In his work, detail electronic effects on pyridones as well as on the aryl halides have been evaluated.

In this report, we noted that $\mathrm{CuI}$ could facilitate the coupling of aryl halides with 2-hydroxypyridines under milder conditions in the presence of $N, N^{\prime}$-dimethylcyclohexane1,2-diamine (DMCDA) as the ligand. The reaction could proceed in toluene instead of dioxane or DMF. ${ }^{12}$ Toluene is a common solvent that could be used for industrial applications.<smiles>N[C@@H]1CCCC[C@H]1N</smiles><smiles>CNC1CCCCC1NC</smiles><smiles>CN(C)CCN(C)C</smiles>

CHDA DMCDA

Recently, we have attempted the synthesis of 2-aminopyridines. ${ }^{13}$ In one experiment, we accidentally observed that 2 equiv of 2-bromopyridine (1) would undergo selfcondensation in the presence of $\mathrm{CuI} / \mathrm{DMCDA} / \mathrm{K}_{2} \mathrm{CO}_{3}$ as the catalyst to give $N$-(pyrid-2-yl)pyrid-2-one (2) as the major product. Although we could not conclusively differentiate the $\mathrm{N}$-arylated or $\mathrm{O}$-arylated structure on the basis of the NMR spectrum, X-ray crystallographic analysis of the selfcondensation product (4) unambiguously shows that the pyridyl group is attached to the pyridone nitrogen atom (Fig. 1, Scheme 1). 


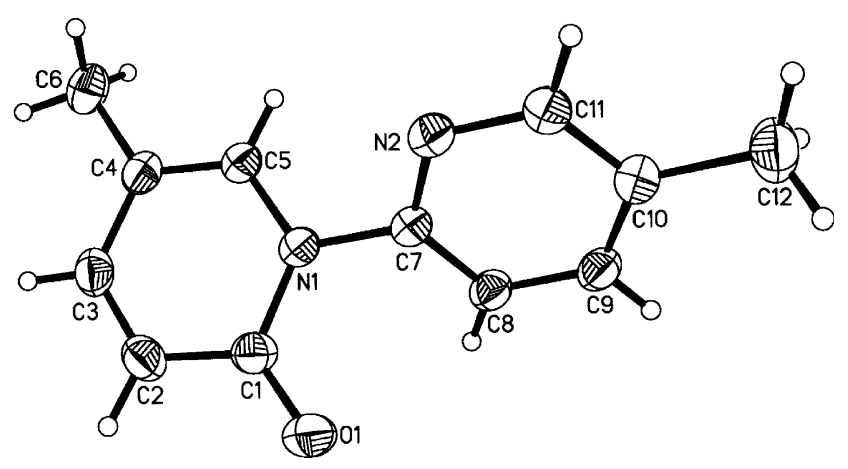

Figure 1. The ORTEP of 4.

2

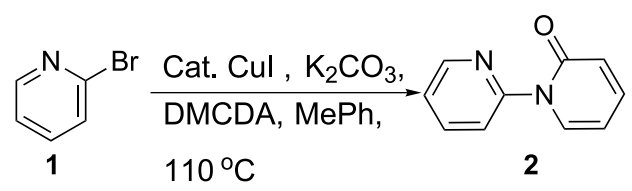

Scheme 1.

It is noteworthy that unsubstituted or tetrasubstituted 1,2diamines such as trans-cyclohexane-1,2-diamine(CHDA) or tetramethylethylene-1,2-diamine (TMEDA) do not promote the self-condensation reaction, indicating that DMCDA is essential for the reaction. When CHDA was applied, no reaction occurred. On the other hand, bromoiodo exchange reaction occurred to give 2-iodopyridine (20\%) in the presence of TMEDA. The self-condensation reaction could be applied to other 2-halopyridines (Table 1).
However, 2-chloropyridine was found to be sluggish in the self-condensation reaction. Although no direct evidence was obtained for the reaction mechanism, we suspect that hydrolysis of 2-bromopyridine (1) under the reaction conditions would occur to give 2-hydroxypyridine. The newly generated 2-hydroxypyridine would then be further coupled with 2 -bromopyridine to give (2). Since we could not isolate any 2-hydroxypyridine from the reaction mixture, we proposed that the coupling of 2-hydroxypyridine with 2-bromopyridine is relatively fast in comparison to the 2-hydroxypyridine formation. These observations suggested that cross coupling of 2-hydroxypyridine with other 2-aryl halides might be feasible under similar reaction conditions (Table 2).

In our initial screening experiments, 2-hydroxypyridine and bromobenzene were used as the prototype substrates for searching the reaction conditions. In contrast to Ukita's reaction conditions ${ }^{4 \mathrm{a}}$ in which no reaction would occur in mesitylene, our reaction proceeds smoothly in toluene at reflux temperature. More surprisingly, the reaction is very specific to 2-hydroxypyridine. Other phenolic derivatives such as 3- and 4-hydroxypyridines, 2-hydroxypyrimidine, and phenol or phthalimide do not couple with bromobenzene under the above conditions. The choice of the ligand strongly affects the reactivity of the cross-coupling reaction. The use of CHDA under similar conditions gave $N$-phenylpyrid-2-one (5) in low yield (3\%). TMEDA does not promote the reaction.

Both $\mathrm{KOAc}$ and $\mathrm{K}_{2} \mathrm{CO}_{3}$ were found to be effective as bases.

Table 1. Self-condensation of 2-halopyridines

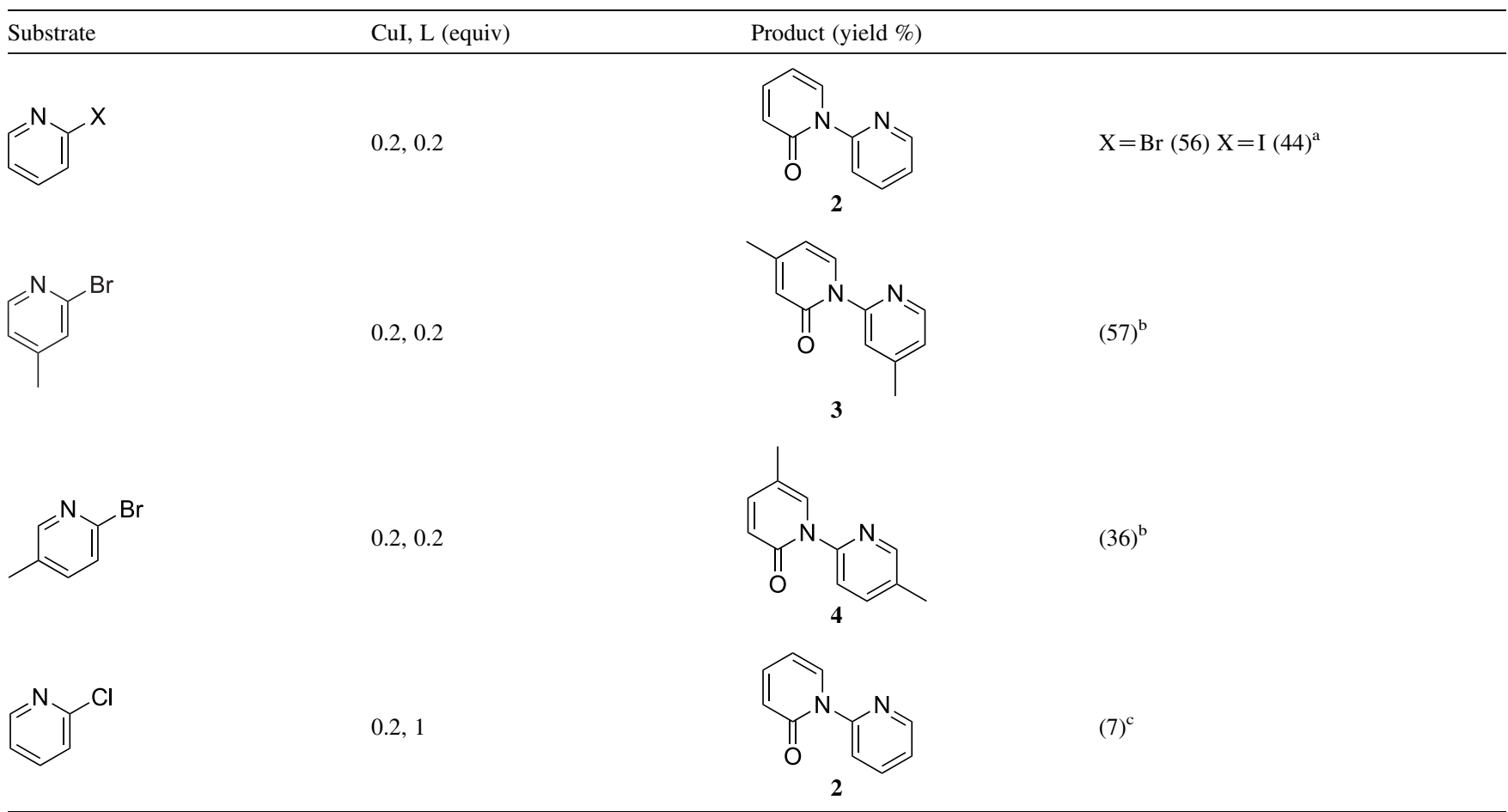

L: trans- $N, N^{\prime}$-Dimethyl-cyclohexane-1,2-diamine.

a $16 \mathrm{~h}$.

b $20 \mathrm{~h}$.

${ }^{c} 24 \mathrm{~h}$. 
Table 2. Cross coupling of 2-hydroxypyridine with various aryl halides

$$
\overbrace{\mathrm{Cul}, \mathrm{DMCDA}, \mathrm{K}_{2} \mathrm{CO}_{3}, \mathrm{PhMe}}^{\mathrm{Arl}}
$$<smiles>[X]c1ccccc1</smiles><smiles>O=c1ccccn1-c1ccc(N(c2ccccc2)c2ccccc2)cc1</smiles>

15

2<smiles>COc1ccc(Br)cc1</smiles><smiles>COc1ccc(-n2ccccc2=O)cc1</smiles>

3<smiles>N#Cc1ccc(Br)cc1</smiles><smiles>Cc1ccc(Br)cc1</smiles><smiles>N#Cc1ccc(-n2ccccc2=O)cc1</smiles>

4<smiles>[H][Z]1ccc(-c2ccccc2)c(=O)n1-c1ccccc1</smiles>

5<smiles>Brc1ccc2ccccc2c1</smiles><smiles>O=c1ccccn1-c1ccc2ccccc2c1</smiles>

6<smiles>C=Cc1ccc(Br)cc1</smiles><smiles>C=Cc1ccc(-n2ccccc2=O)cc1</smiles>

11<smiles>COc1ccccc1Br</smiles><smiles>COc1ccccc1-n1ccccc1=O</smiles><smiles>COc1ccc(-n2ccccc2=O)cc1Br</smiles><smiles>COc1ccc(Br)cc1Br</smiles>

10<smiles>Brc1cccc2ccccc12</smiles><smiles>O=c1ccccn1-c1cccc2ccccc12</smiles>

14
(70)

(89)

(61)

(82)

(38)

12<smiles>Br[Z20]c1ccc(N(c2ccccc2)c2ccccc2)cc1</smiles>

13<smiles>Brc1ccccn1</smiles>

14<smiles>Brc1cccnc1</smiles>

15<smiles>Brc1cccc(Br)n1</smiles>

16<smiles>Brc1nccs1</smiles>

$(2)^{a}$

(37)

(81)<smiles>Cc1cc(-n2ccccc2=O)ccc1N(c1ccccc1)c1ccccc1</smiles>

16<smiles>O=c1ccccn1-c1ccccn1</smiles>

17<smiles>O=c1ccccn1-c1c[13c]2cccc(=O)n2c2cccc(=O)n12</smiles>

17<smiles>Brc1ccsc1</smiles>

18<smiles>O=[N+]([O-])c1cccc(Br)c1</smiles><smiles>O=c1ccccn1-c1nccs1</smiles><smiles>O=c1ccccn1-c1ccsc1</smiles>

20<smiles>O=c1ccccn1-c1cccc([N+](=O)[O-])c1</smiles>

21<smiles>CSc1ccc(-n2ccccc2=O)cc1</smiles> 
However, $\mathrm{KO}^{t} \mathrm{Bu}$ will attack 2-bromopyridine to give 2-tert-butoxypyridine. ${ }^{14}$ The reaction will also proceed in other solvents such as DMF or dioxane.

However, the use of toluene gave the best results. The optimized reaction conditions of $20 \mathrm{~mol} \% \mathrm{CuI}, 20 \mathrm{~mol} \%$ DMCDA, and 2 equiv $\mathrm{K}_{2} \mathrm{CO}_{3}$ were applied to the $N$-arylation of 2-hydroxypyridine with a number of aryl halides (Table 2). Functional groups that are compatible with this CuI-catalyzed $N$-arylation protocol include ether, thioether, triarylamine, nitrile, nitro group, styrene, and ester. No significant electronic effects on the aryl halides were observed in the reaction. As can be seen, the electrondeficient aryl bromides such as $3-\mathrm{NO}_{2} \mathrm{C}_{6} \mathrm{H}_{4} \mathrm{Br}$ and $4-\mathrm{CNC}_{6}$ $\mathrm{H}_{4} \mathrm{Br}$, as well as the electron-rich aryl bromides such as 4- $\mathrm{MeOC}_{6} \mathrm{H}_{4} \mathrm{Br}$ or $\mathrm{Ph}_{2} \mathrm{NC}_{6} \mathrm{H}_{4} \mathrm{Br}$ are effectively coupled in the reaction.

Electronic effects on 2-hydroxypyridine are significant, however. Electron-withdrawing substituents that would reduce the nucleophilicity of 2-hydroxypyridine do retard its reactivity toward the coupling reaction. Thus, the reaction of 2-hydroxy-5-nitropyridine is very sluggish. Similar results were reported in Li's conditions.

The reaction could also be applied to heterocyclic aryl halides such as pyridine, thiophene, and thiazole. In contrast to the results of Ukita's and Li's conditions, our conditions could also be applied to 3-bromopyridine to give the corresponding pyridone $\mathbf{1 7}$ in high yield.

The reaction is very sensitive to the steric environment of the aryl halides. Usually, $o$-substituted aryl bromides do not show enough reactivity in the reaction. Thus, regioselective coupling would occur at the 4-position of 2,4-dibromoanisole to give the para substituted product (entry 9, Table 2).

Similarly, although 2-bromonaphthalene could react smoothly to give the desired pyridone, as mentioned in Ukita's paper, 1-bromonaphthalene does not react under these conditions (compare entries 5 and 10, Table 2). We have applied this method for purifying 1-bromonaphthalene. Commercially available 1-bromonaphthalene is usually contaminated by a few percent of 2-bromonaphthalene. Since they have similar polarity and boiling point, they are difficult to separate. When the contaminated 1-bromonaphthalene was treated with 2-hydroxypyridine in the presence of the copper catalyst, the 2-bromo impurity was selectively converted to the more polar pyridone and removed by liquid chromatography. No bromo-iodo exchange was observed under these reaction conditions.

The reactivity of the reaction is so high that it could be successfully applied to polymer modification. Treatment of the commercially available poly(4-bromostyrene) (23) with 2-hydroxypyridine under the same conditions affords the target polypyridone (24) in reasonable yield. The structural assignment was supported by IR and NMR spectroscopy. The final polymer shows characteristic IR absorption bands of pyridone at 1663 and $1590 \mathrm{~cm}^{-1}$, along with the disappearance of the characteristic IR absorption band of poly(4-bromostyrene) at $1073 \mathrm{~cm}^{-1}$. In addition, five aromatic ${ }^{1} \mathrm{H}$ NMR signals at $\delta 6.14(1 \mathrm{H}), 6.42(1 \mathrm{H}), 6.81$ $(2 \mathrm{H}), 7.23(2 \mathrm{H})$, and $7.41(2 \mathrm{H})$, and nine sets of $\mathrm{sp}^{2}{ }^{13} \mathrm{C}$ NMR signals at $\delta 105.2,120.3,125.9,127.6,138.2,138.7$, $140.0,144.9$ and 160.8 suggested the polypyridone formation (Scheme 2).<smiles>CCC(C)C(C)(C)C</smiles>

23

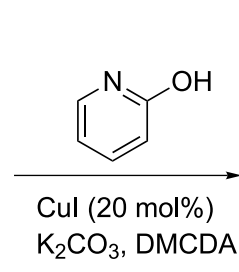

$\mathrm{K}_{2} \mathrm{CO}_{3}$, DMCDA

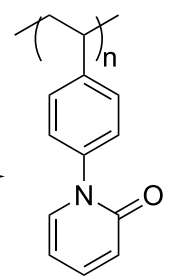

$24(56 \%)$
Scheme 2 .

The reaction could be extended to iterative oligo-pyridone synthesis (Scheme 3). As mentioned before, the bromo group of 2-bromopyridine could be replaced by tertbutoxide in the presence of $\mathrm{KO}^{t} \mathrm{Bu}$. Thus, reaction of $\mathbf{2 5}$ with $\mathrm{KO}^{t} \mathrm{Bu}$ afforded 26. CuI-catalyzed condensation of 2hydroxypyridine with $\mathbf{2 6}$ gave $\mathbf{2 7}$ in moderated yield.
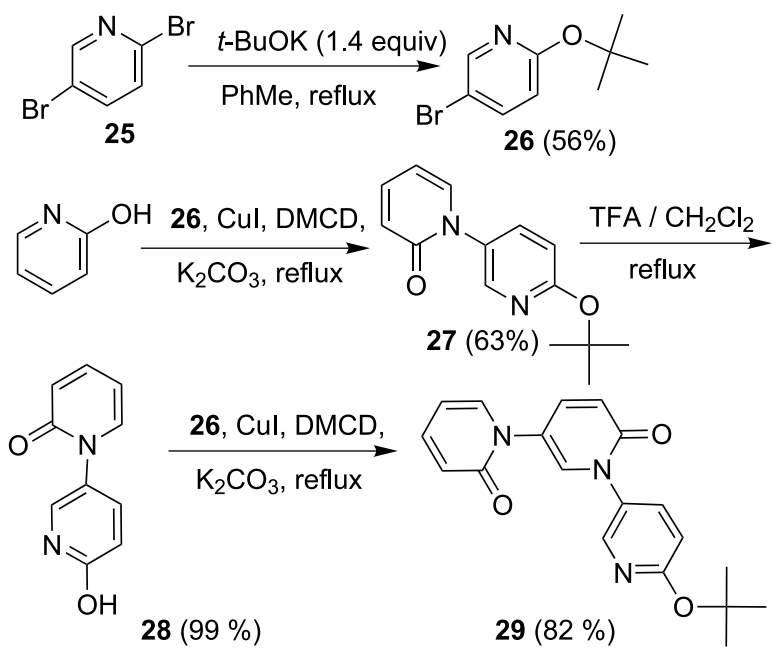<smiles>[Z7][13CH2]NC(C)(C)O</smiles>

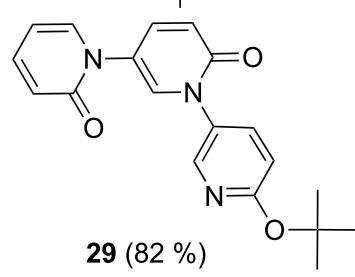

Scheme 3.

Deprotection of $\mathbf{2 7}$ by removal of the tert-butyl group in TFA afforded $\mathbf{2 8}$ in high yield. Compound $\mathbf{2 8}$ is slightly hygroscopic and has to be dried under vacuum at $110^{\circ} \mathrm{C}$ before use in order to obtain the third generation of oligopyridone 29 in high yield. This iterative approach proved to be effective. Further application of this approach to the synthesis of tailored oligo-pyridones is ongoing (Scheme 3).

\section{Experimental}

\subsection{The CuI-catalyzed self-condensation of 2-bromo- pyridine}

trans- $N, N^{\prime}$-Dimethylcyclohexane-1,2-diamine (DMCDA) 
was prepared according to the supporting information in literature. $^{9 \mathrm{c}}$

\subsection{General procedure}

To an oven-dried double-necked flask containing a stir-bar was charged $\mathrm{CuI}(20 \mathrm{~mol} \%)$ and $\mathrm{K}_{2} \mathrm{CO}_{3}$ (2 equiv). The flask was evacuated and backfilled with nitrogen. A solution of 2-bromopyridine in toluene (1 M, 1 equiv) and DMCDA $(20 \mathrm{~mol} \%)$ were injected under nitrogen. The reaction mixture was stirred and heated at reflux temperature for the time specified. The resulting mixture was cooled to room temperature, diluted with dichloromethane, and filtered. The filtrate was washed with water. The organic phase was collected, dried over anhydrous $\mathrm{MgSO}_{4}$, and concentrated. The crude product was purified by flash chromatography on silica gel (ethyl acetate/dichloromethane).

2.2.1. 1-(2-Pyridyl)-1H-pyridin-2-one (2). ${ }^{15}$ Reaction time: $16 \mathrm{~h}$, white solid (56\%). Mp 53-54 ${ }^{\circ} \mathrm{C}$ (from hexane); ${ }^{1} \mathrm{H}$ NMR $\left(400 \mathrm{MHz}^{\mathrm{CDCl}}{ }_{3}\right) \delta 8.54(\mathrm{~d}, J=5.2 \mathrm{~Hz}, 1 \mathrm{H}), 7.91$ $(\mathrm{d}, J=6.8 \mathrm{~Hz}, 1 \mathrm{H}), 7.79-7.84(\mathrm{~m}, 2 \mathrm{H}), 7.36(\mathrm{ddd}, J=9.2$, $6.8,2.4 \mathrm{~Hz}, 1 \mathrm{H}), 7.29$ (ddd, $J=6.8,5.2,1.2 \mathrm{~Hz}, 1 \mathrm{H}), 6.61$ $(\mathrm{d}, J=9.2 \mathrm{~Hz}, 1 \mathrm{H}), 6.27(\mathrm{td}, J=6.8,1.2 \mathrm{~Hz}, 1 \mathrm{H}) ;{ }^{13} \mathrm{C} \mathrm{NMR}$ $\left(100 \mathrm{MHz}, \mathrm{CDCl}_{3}\right) \delta 161.9,151.5,148.6,140.0,137.5$, 135.8, 123.0, 121.8, 121.2, 106.1; IR (KBr): 3058, 2991, $1673(\mathrm{C}=\mathrm{O}), 1612,1540 \mathrm{~cm}^{-1}$; FAB (NBA) $173.1{\left(\mathrm{M}^{+}+\right.}^{+}$ $\mathrm{H})$; HRMS calcd for $\mathrm{C}_{10} \mathrm{H}_{9} \mathrm{~N}_{2} \mathrm{O} 173.0715$, found 173.0712 $\left(\mathrm{M}^{+}+\mathrm{H}\right)$, calcd for $\mathrm{C}_{10} \mathrm{H}_{8} \mathrm{~N}_{2} \mathrm{O} 172.0637$, found 172.0639 $\left(\mathrm{M}^{+}\right)$. Anal. calcd for $\mathrm{C}_{10} \mathrm{H}_{8} \mathrm{~N}_{2} \mathrm{O} \mathrm{C}, 69.76 ; \mathrm{H}, 4.68 ; \mathrm{N}$, 16.27. Found C, 69.73; H, 4.70; N, 16.26.

2.2.2. 1-(4-Methylpyrid-2-yl)-4-methyl-1H-pyridin-2one (3). ${ }^{15}$ Reaction time: $20 \mathrm{~h}$, colorless solid (57\%). Mp 79-81 ${ }^{\circ} \mathrm{C}$ (from hexane); ${ }^{1} \mathrm{H}$ NMR (400 MHz, $\left.\mathrm{CDCl}_{3}\right) \delta$ $8.37(\mathrm{~d}, J=5.2 \mathrm{~Hz}, 1 \mathrm{H}), 7.70-7.72(\mathrm{~m}, 2 \mathrm{H}), 7.09$ (ddd, $J=$ $5.2,1.2,0.8 \mathrm{~Hz}, 1 \mathrm{H}), 6.41$ (bs, $1 \mathrm{H}), 6.10(\mathrm{dd}, J=7.2,2 \mathrm{~Hz}$, $1 \mathrm{H}), 2.40(\mathrm{~s}, 3 \mathrm{H}), 2.20(\mathrm{~d}, J=0.8 \mathrm{~Hz}, 3 \mathrm{H}) ;{ }^{13} \mathrm{C} \mathrm{NMR}$ $\left(100 \mathrm{MHz}, \mathrm{CDCl}_{3}\right) \delta 161.9,151.8,151.7,149.1,148.2$, 134.9, 124.0, 121.9, 119.9, 108.8, 21.4, 21.3; IR $\left(\mathrm{CH}_{2} \mathrm{Cl}_{2}\right)$ : 3044, 2922, $1670(\mathrm{C}=\mathrm{O}), 1614,1599,1537,1406 \mathrm{~cm}^{-1}$; FAB (NBA) $201.1\left(\mathrm{M}^{+}+\mathrm{H}\right), 200.1\left(\mathrm{M}^{+}\right)$; HRMS calcd for $\mathrm{C}_{12} \mathrm{H}_{13} \mathrm{~N}_{2} \mathrm{O} 201.1028$, found $201.1031\left(\mathrm{M}^{+}+\mathrm{H}\right)$, calcd for $\mathrm{C}_{12} \mathrm{H}_{12} \mathrm{~N}_{2} \mathrm{O} 200.0950$, found $200.0951\left(\mathrm{M}^{+}\right)$. Anal. calcd for $\mathrm{C}_{12} \mathrm{H}_{12} \mathrm{~N}_{2} \mathrm{O}$ C, 71.98; H, 6.04; N, 13.99. Found C, 71.89; H, 6.09; N, 13.98 .

2.2.3. 1-(5-Methylpyrid-2-yl)-5-methyl-1H-pyridin-2one (4). ${ }^{16}$ Reaction time: $24 \mathrm{~h}$, colorless solid (36\%). Mp 106-107 ${ }^{\circ} \mathrm{C}$ (from hexane); ${ }^{1} \mathrm{H}$ NMR (400 MHz, $\left.\mathrm{CD}_{2} \mathrm{Cl}_{2}\right) \delta$ 8.36 (bs, $1 \mathrm{H}), 7.72$ (d, $J=8 \mathrm{~Hz}, 1 \mathrm{H}), 7.63$ (dd, $J=8,2 \mathrm{~Hz}$, $1 \mathrm{H}), 7.58(\mathrm{dd}, J=2.4,0.8 \mathrm{~Hz}, 1 \mathrm{H}), 7.25(\mathrm{dd}, J=9.2,2.4 \mathrm{~Hz}$, $1 \mathrm{H}), 6.48(\mathrm{~d}, J=9.6 \mathrm{~Hz}, 1 \mathrm{H}), 2.38(\mathrm{~s}, 3 \mathrm{H}), 2.11(\mathrm{~d}, J=$ $1.2 \mathrm{~Hz}, 1 \mathrm{H}) ;{ }^{13} \mathrm{C}$ NMR $\left(100 \mathrm{MHz}, \mathrm{CDCl}_{3}\right) \delta 161.4,149.7$, 148.7, 142.7, 138.1, 133.1, 132.7, 121.4, 120.7, 115.0, 18.1, 17.3; IR $\left(\mathrm{CH}_{2} \mathrm{Cl}_{2}\right): 3047,2926,1681(\mathrm{C}=\mathrm{O}), 1614,1593$, 1530, $1498 \mathrm{~cm}^{-1}$; FAB (NBA) $201.1\left(\mathrm{M}^{+}+\mathrm{H}\right), 200.1$ $\left(\mathrm{M}^{+}\right)$; HRMS calcd for $\mathrm{C}_{12} \mathrm{H}_{13} \mathrm{~N}_{2} \mathrm{O}$ 201.1028, found $201.1026\left(\mathrm{M}^{+}+\mathrm{H}\right)$, calcd for $\mathrm{C}_{12} \mathrm{H}_{12} \mathrm{~N}_{2} \mathrm{O} 200.0950$, found $200.0946\left(\mathrm{M}^{+}\right)$. Anal. calcd for $\mathrm{C}_{12} \mathrm{H}_{12} \mathrm{~N}_{2} \mathrm{O} \mathrm{C}$, 71.98; H, 6.04; N, 13.99. Found C, 71.97; H, 6.07; N, 13.98.

\subsection{General procedure for the CuI-catalyzed $N$ - arylation of 2-hydroxypyridine}

To an oven-dried double-necked flask containing a stir-bar was charged 2-hydroxypyridine (1 equiv), CuI (20 mol\%), and $\mathrm{K}_{2} \mathrm{CO}_{3}$ (2 equiv). The flask was evacuated and backfilled with nitrogen. A solution of arylbromide in toluene (1 M, 1 equiv) and DMCDA (20 mol\%) were injected under nitrogen. The reaction mixture was stirred and heated at reflux temperature for the time specified. The resulting mixture was cooled to room temperature, diluted with dichloromethane, and filtered. The filtrate was washed with water. The organic phase was collected, dried over anhydrous $\mathrm{MgSO}_{4}$, and concentrated. The crude product was purified by flash chromatography on silica gel (ethyl acetate/dichloromethane).

2.3.1. 1-Phenyl-1H-pyridin-2-one (5). ${ }^{17}$ Reaction time: $18 \mathrm{~h}$, colourless solid (91\%). Mp $153-154{ }^{\circ} \mathrm{C}$ (from $\mathrm{CH}_{2} \mathrm{Cl}_{2} /$ hexane $=1: 10) ;{ }^{1} \mathrm{H}$ NMR $\left(400 \mathrm{MHz}, \mathrm{d}_{6}\right.$-DMSO) $\delta 7.62(\mathrm{dd}$, $J=7.2,1.6 \mathrm{~Hz}, 1 \mathrm{H}), 7.47-7.52(\mathrm{~m}, 3 \mathrm{H}), 7.44(\mathrm{~d}, J=7.2 \mathrm{~Hz}$, $1 \mathrm{H}), 7.33-7.41(\mathrm{~m}, 2 \mathrm{H}), 6.47(\mathrm{~d}, J=8.8 \mathrm{~Hz}, 1 \mathrm{H}), 6.30(\mathrm{td}$, $J=6.8,1.2 \mathrm{~Hz}, 1 \mathrm{H}) ;{ }^{13} \mathrm{C} \mathrm{NMR}\left(100 \mathrm{MHz} \mathrm{CDCl}_{3}\right) \delta 162.1$, 140.7, 139.6, 137.8, 129.1, 128.3, 126.3, 121.7, 105.8; IR (KBr): 3038, 1671(C=O), 1611, 1585, 1499, $1447 \mathrm{~cm}^{-1}$; FAB (NBA) $172.1\left(\mathrm{M}^{+}+\mathrm{H}\right)$, HRMS calcd for $\mathrm{C}_{11} \mathrm{H}_{10} \mathrm{NO}$ 171.0762, found $172.0759\left(\mathrm{M}^{+}+\mathrm{H}\right)$, calcd for $\mathrm{C}_{11} \mathrm{H}_{9} \mathrm{NO}$ 171.0684, found $171.0686\left(\mathrm{M}^{+}\right)$. Anal. calcd for $\mathrm{C}_{11} \mathrm{H}_{9} \mathrm{NO}$ : C, 77.17; H, 5.30; N, 8.18. Found C, 77.30; H, 5.12; N, 8.11.

2.3.2. 1-(4-Methoxyphenyl)-1H-pyrid-2-one (6). Reaction time: $20 \mathrm{~h}$, white solid $(89 \%)$. Mp $110-111^{\circ} \mathrm{C}$ (from $\mathrm{CH}_{2} \mathrm{Cl}_{2}$ /hexane $\left.=1: 10\right) ;{ }^{1} \mathrm{H} \mathrm{NMR}$ (400 MHz, $\mathrm{d}_{6}$-acetone) $\delta$ 7.5 (ddd, $J=7,2.2,0.8 \mathrm{~Hz}, 1 \mathrm{H}), 7.43$ (ddd, $J=9.2,6.7$, $2.2 \mathrm{~Hz}, 1 \mathrm{H}), 7.32(\mathrm{dt}, J=9.2,2.8 \mathrm{~Hz}, 2 \mathrm{H}), 7.02(\mathrm{dt}, J=9.2$, $2.8 \mathrm{~Hz}, 2 \mathrm{H}), 6.42(\mathrm{ddd}, J=9.2,1.2,0.8 \mathrm{~Hz}, 1 \mathrm{H}), 6.24(\mathrm{td}$, $J=6.7,1.6 \mathrm{~Hz}, 1 \mathrm{H}), 3.85(\mathrm{~s}, 3 \mathrm{H}) ;{ }^{13} \mathrm{C} \mathrm{NMR}(100 \mathrm{MHz}$, $\left.\mathrm{CDCl}_{3}\right) \delta 162.4,159.1,139.5,138.1,133.6,127.4,121.6$, 114.4, 105.6, 55.5; IR (KBr): 3024, 2363, 2325, 1661 $(\mathrm{C}=\mathrm{O}), 1599,1499,1435 \mathrm{~cm}^{-1}$; FAB (NBA) 202.1 $\left(\mathrm{M}^{+}+\mathrm{H}\right)$; HRMS calcd for $\mathrm{C}_{12} \mathrm{H}_{12} \mathrm{NO}_{2} 202.0868$, found $202.0865\left(\mathrm{M}^{+}+\mathrm{H}\right)$, calcd for $\mathrm{C}_{12} \mathrm{H}_{11} \mathrm{NO}_{2} 201.0790$, found 201.0797 $\left(\mathrm{M}^{+}\right)$. Anal. calcd for $\mathrm{C}_{12} \mathrm{H}_{11} \mathrm{NO}_{2}$ : C, 71.63; H, 5.51; N, 6.96. Found C, 71.93; H, 5.29; N, 6.87.

2.3.3. 1-(4-Cyanophenyl)-1H-pyridin-2-one (7). ${ }^{18}$ Reaction time: $12 \mathrm{~h}$, white solid $(70 \%)$. Mp $165-166^{\circ} \mathrm{C}$ (from $\mathrm{CH}_{2} \mathrm{Cl}_{2}$ /hexane $\left.=1: 10\right) ;{ }^{1} \mathrm{H}$ NMR $\left(400 \mathrm{MHz}, \mathrm{CDCl}_{3}\right) \delta 7.75$ $(\mathrm{d}, J=8.4 \mathrm{~Hz}, 2 \mathrm{H}), 7.51(\mathrm{~d}, J=8.4 \mathrm{~Hz}, 2 \mathrm{H}), 7.39$ (ddd, $J=$ 9.2, 6.8, 2.2 Hz, $1 \mathrm{H}), 7.28(\mathrm{dd}, J=7,2.2 \mathrm{~Hz}, 1 \mathrm{H}), 6.61(\mathrm{~d}$, $J=9.2 \mathrm{~Hz}, 1 \mathrm{H}), 6.26(\mathrm{t}, J=6.8 \mathrm{~Hz}, 1 \mathrm{H}) ;{ }^{13} \mathrm{C} \mathrm{NMR}$ $\left(100 \mathrm{MHz}, \mathrm{CDCl}_{3}\right) \delta 161.5,144.1,140.1,136.5,133.0$, 127.3, 121.9, 117.7, 112.1, 106.6; IR (KBr): 3100, 3038, 2362, 2325, $1673(\mathrm{C}=\mathrm{O}), 1599,1523,1499 \mathrm{~cm}^{-1}$; FAB (NBA) $197.0\left(\mathrm{M}^{+}+\mathrm{H}\right)$; HRMS calcd for $\mathrm{C}_{12} \mathrm{H}_{9} \mathrm{~N}_{2} \mathrm{O}$ 197.0715, found $197.0711\left(\mathrm{M}^{+}+\mathrm{H}\right)$, calcd for $\mathrm{C}_{12} \mathrm{H}_{8} \mathrm{~N}_{2} \mathrm{O}$ 196.0637, found $196.0643\left(\mathrm{M}^{+}\right)$. Anal. calcd for $\mathrm{C}_{12} \mathrm{H}_{8} \mathrm{~N}_{2} \mathrm{O}$ : C, 73.46; H, 4.11; N, 14.28. Found C, 73.28; $\mathrm{H}, 3.98 ; \mathrm{N}, 14.16$.

2.3.4. 1-(Biphenyl-4-yl)-1H-pyridin-2-one (8). ${ }^{19}$ Reaction time: $14 \mathrm{~h}$, white solid (38\%). Mp 226-227 ${ }^{\circ} \mathrm{C}$ (from $\mathrm{CH}_{2} \mathrm{Cl}_{2}$ /hexane $\left.=1: 10\right) ;{ }^{1} \mathrm{H} \mathrm{NMR}\left(400 \mathrm{MHz}, \mathrm{CDCl}_{3}\right) \delta$ 
$7.68(\mathrm{dd}, J=6.6,1.8 \mathrm{~Hz}, 2 \mathrm{H}), 7.58(\mathrm{~d}, J=9.2 \mathrm{~Hz}, 2 \mathrm{H})$, $7.34-7.46(\mathrm{~m}, 7 \mathrm{H}), 6.68(\mathrm{~d}, J=9.2 \mathrm{~Hz}, 1 \mathrm{H}), 6.29(\mathrm{td}, J=$ 6.6, $1.2 \mathrm{~Hz}, 1 \mathrm{H}) ;{ }^{13} \mathrm{C}$ NMR $\left(100 \mathrm{MHz}, \mathrm{CDCl}_{3}\right) \delta 162.2$, $141.4,139.9,139.8,139.7,137.7,128.7,127.9,127.6$, 127.1, 126.6, 121.7, 106.0; IR (KBr): 3062, 3024, 2363, 2337, $1661(\mathrm{C}=\mathrm{O}), 1585,1535,1485 \mathrm{~cm}^{-1}$; FAB (NBA) $248.1\left(\mathrm{M}^{+}+\mathrm{H}\right)$; HRMS calcd for $\mathrm{C}_{17} \mathrm{H}_{14} \mathrm{NO} 248.1075$, found $248.1074\left(\mathrm{M}^{+}+\mathrm{H}\right)$, calcd for $\mathrm{C}_{17} \mathrm{H}_{13} \mathrm{NO} 247.0997$, found $247.0993\left(\mathrm{M}^{+}\right)$. Anal. calcd for $\mathrm{C}_{17} \mathrm{H}_{13} \mathrm{NO}$ : C, 82.57; H, 5.30; N, 5.66. Found C, 82.26; H,5.17; N, 5.46.

2.3.5. 1-(Naphthalen-2-yl)-1H-pyridin-2-one (9). Reaction time: $18 \mathrm{~h}$, white solid, (82\%). Mp $160-161^{\circ} \mathrm{C}$ (from $\mathrm{CH}_{2} \mathrm{Cl}_{2} /$ hexane $\left.=1: 10\right) ;{ }^{1} \mathrm{H}$ NMR $\left(400 \mathrm{MHz}, \mathrm{CDCl}_{3}\right) \delta 7.92$ $(\mathrm{d}, J=8.8 \mathrm{~Hz}, 1 \mathrm{H}), 7.81-7.89(\mathrm{~m}, 2 \mathrm{H}), 7.79(\mathrm{~d}, J=2 \mathrm{~Hz}$, $1 \mathrm{H}), 7.48-7.54(\mathrm{~m}, 3 \mathrm{H}), 7.37-7.43(\mathrm{~m}, 2 \mathrm{H}), 6.68(\mathrm{dd}, J=$ $9.8,1.4 \mathrm{~Hz}, 1 \mathrm{H}), 6.25(\mathrm{td}, J=6.8,0.8 \mathrm{~Hz}, 1 \mathrm{H}) ;{ }^{13} \mathrm{C}$ NMR $\left(100 \mathrm{MHz}, \mathrm{CDCl}_{3}\right) \delta 162.3,139.7,138.5,138.0,133.2$, $132.6,129.0,127.9,127.6,126.7,126.6,124.7,124.4$, 121.8, 105.9; IR (KBr): 3024, 2362, 2337, $1661(\mathrm{C}=\mathrm{O})$, $1585,1535,1499,1461 \mathrm{~cm}^{-1}$; FAB (NBA) $222.1\left(\mathrm{M}^{+}+\right.$ $\mathrm{H}), 221.1\left(\mathrm{M}^{+}\right)$; HRMS calcd for $\mathrm{C}_{15} \mathrm{H}_{12} \mathrm{NO} 222.0919$, found $222.0921\left(\mathrm{M}^{+}+\mathrm{H}\right)$, calcd for $\mathrm{C}_{15} \mathrm{H}_{11} \mathrm{NO} 221.0841$, found $221.0841\left(\mathrm{M}^{+}\right)$. Anal. calcd for $\mathrm{C}_{15} \mathrm{H}_{11} \mathrm{NO}$ : C, 81.43; H, 5.01; N, 6.33. Found C, 81.16; H, 5.11; N, 6.31.

2.3.6. 1-(4-Vinylphenyl)-1H-pyridin-2-one (10). Reaction time: $9 \mathrm{~h}$, white solid $(61 \%)$. Mp $115-116^{\circ} \mathrm{C}$ (from $\mathrm{CH}_{2} \mathrm{Cl}_{2} /$ hexane $=1: 10) ;{ }^{1} \mathrm{H} \mathrm{NMR}\left(400 \mathrm{MHz}, \mathrm{CDCl}_{3}\right) \delta 7.49(\mathrm{dt}, J=$ $8.4,2 \mathrm{~Hz}, 2 \mathrm{H}), 7.36$ (ddd, $J=9.2,6.8,2.4 \mathrm{~Hz}, 1 \mathrm{H}), 7.28$ $7.34(\mathrm{~m}, 3 \mathrm{H}), 6.72(\mathrm{dd}, J=17.6,10.8 \mathrm{~Hz}, 1 \mathrm{H}), 6.63(\mathrm{~d}, J=$ $9.2 \mathrm{~Hz}, 1 \mathrm{H}), 6.21(\mathrm{td}, J=6.8,1.6 \mathrm{~Hz}, 1 \mathrm{H}), 5.77(\mathrm{dd}, J=$ $17.6,0.8 \mathrm{~Hz}, 1 \mathrm{H}), 5.30(\mathrm{dd}, J=10.8,0.8 \mathrm{~Hz}, 1 \mathrm{H}),{ }^{13} \mathrm{C} \mathrm{NMR}$ $\left(100 \mathrm{MHz}, \mathrm{CDCl}_{3}\right)$ only 10 sets of ${ }^{13} \mathrm{C}$ signals was observed $\delta 162.1,140.0,139.6,137.6,135.5,126.9,126.4,121.8$, 115.2, 105.8; IR (KBr): 3029, $1668(\mathrm{C}=\mathrm{O}), 1582,1530$, 1490, $1455 \mathrm{~cm}^{-1}$; FAB (NBA) $198.1\left(\mathrm{M}^{+}+\mathrm{H}\right), 197.1$ $\left(\mathrm{M}^{+}\right)$; HRMS calcd for $\mathrm{C}_{13} \mathrm{H}_{12} \mathrm{NO} 198.0919$, found 198.0918 $\left(\mathrm{M}^{+}+\mathrm{H}\right)$, calcd for $\mathrm{C}_{13} \mathrm{H}_{11} \mathrm{NO} 197.0841$, found 197.0839 $\left(\mathrm{M}^{+}\right)$. Anal. calcd for $\mathrm{C}_{13} \mathrm{H}_{11} \mathrm{NO} \mathrm{C}, 79.17 ; \mathrm{H}$, 5.62; N, 7.10. Found C, 79.33; H, 5.64; N, 7.28.

\subsection{7. $4,4^{\prime}$-Bis(2-oxo-2H-pyridin-1-yl)biphenyl-2,2'} dicarboxylic acid dimethyl ester (11). Reaction time: $16 \mathrm{~h}$, white solid (81\%). Mp 218-219 ${ }^{\circ} \mathrm{C}$ (from $\mathrm{CH}_{3} \mathrm{CN}$ ); ${ }^{1} \mathrm{H}$ NMR $\left(400 \mathrm{MHz}, \mathrm{CDCl}_{3}\right) \delta 8.07(\mathrm{~d}, J=2.4 \mathrm{~Hz}, 2 \mathrm{H}), 7.63$ $(\mathrm{dd}, J=8,2 \mathrm{~Hz}, 2 \mathrm{H}), 7.40-7.46(\mathrm{~m}, 4 \mathrm{H}), 7.37(\mathrm{~d}, J=8.4 \mathrm{~Hz}$, $2 \mathrm{H}), 6.68(\mathrm{~d}, J=9.2 \mathrm{~Hz}, 2 \mathrm{H}), 6.29(\mathrm{t}, J=6.8 \mathrm{~Hz}, 2 \mathrm{H}), 3.66$ $(\mathrm{s}, 6 \mathrm{H}) ;{ }^{13} \mathrm{C}$ NMR $\left(100 \mathrm{MHz}, \mathrm{CDCl}_{3}\right) \delta 165.7,162.0,142.3$, $139.9,139.7,137.4,131.0,130.0,129.6,127.8,121.8$, 106.2, 52.1; IR (KBr): 3626, 3450, 3062, 3038, 2951, 2363, 2336, $1737(\mathrm{C}=\mathrm{O}), 1661(\mathrm{C}=\mathrm{O}), 1585,1523,1473 \mathrm{~cm}^{-1}$; FAB (NBA) 457.1 $\left(\mathrm{M}^{+}+\mathrm{H}\right), 456.1\left(\mathrm{M}^{+}\right)$; HRMS calcd for $\mathrm{C}_{26} \mathrm{H}_{21} \mathrm{~N}_{2} \mathrm{O}_{6} 457.1400$, found $457.1408\left(\mathrm{M}^{+}+\mathrm{H}\right)$, calcd for $\mathrm{C}_{26} \mathrm{H}_{20} \mathrm{~N}_{2} \mathrm{O}_{6} 456.1321$, found $456.1326\left(\mathrm{M}^{+}\right)$. Anal. calcd for $\mathrm{C}_{26} \mathrm{H}_{20} \mathrm{~N}_{2} \mathrm{O}_{6} \mathrm{C}, 68.42 ; \mathrm{H}, 4.42 ; \mathrm{N}, 6.14$. Found $\mathrm{C}, 68.17$; $\mathrm{H}, 4.34 ; \mathrm{N}, 6.17$.

2.3.8. 1-(2-Methoxyphenyl)- $1 H$-pyridin-2-one $(12) .^{20}$ Reaction time: $16 \mathrm{~h}$ (in DMF), white solid (2\%). Mp 86$87^{\circ} \mathrm{C}$ (from $\mathrm{Et}_{2} \mathrm{O}$ ); ${ }^{1} \mathrm{H}$ NMR $\left(400 \mathrm{MHz}, \mathrm{CDCl}_{3}\right) \delta 7.35$ $7.41(\mathrm{~m}, 2 \mathrm{H}), 7.25(\mathrm{dd}, J=7.6,1.6 \mathrm{~Hz}, 1 \mathrm{H}), 7.18(\mathrm{dd}, J=$ 7.6, $1.6 \mathrm{~Hz}, 1 \mathrm{H}), 7.01-7.05(\mathrm{~m}, 2 \mathrm{H}), 6.64(\mathrm{~d}, J=9.2 \mathrm{~Hz}$,
$1 \mathrm{H}), 6.18(\mathrm{td}, J=6.4,1.2 \mathrm{~Hz}, 1 \mathrm{H}), 3.80(\mathrm{~s}, 3 \mathrm{H}) ;{ }^{13} \mathrm{C} \mathrm{NMR}$ $\left(100 \mathrm{MHz}, \mathrm{CDCl}_{3}\right) \delta 162.2,154.0,139.7,138.8,130.1$, $129.5,128.4,121.7,120.8,112.3,105.3,55.9$; IR (neat): 3062, 2989, $1661(\mathrm{C}=\mathrm{O}), 1599,1523,1485 \mathrm{~cm}^{-1} ; \mathrm{FAB}$ (NBA) $202.0\left(\mathrm{M}^{+}+\mathrm{H}\right)$; HRMS calcd for $\mathrm{C}_{12} \mathrm{H}_{12} \mathrm{NO}_{2}$ 202.0868, found $202.0868\left(\mathrm{M}^{+}+\mathrm{H}\right)$, calcd for $\mathrm{C}_{12} \mathrm{H}_{11} \mathrm{NO}_{2} 201.0790$, found $201.0784\left(\mathrm{M}^{+}\right)$.

2.3.9. 1-(3-Bromo-4-methoxyphenyl)pyridin-2-one (13). Reaction time: $14 \mathrm{~h}$, white solid (37\%). Mp $146-147^{\circ} \mathrm{C}$ (from THF); ${ }^{1} \mathrm{H}$ NMR (400 MHz, $\mathrm{d}_{6}$-DMSO) $\delta 7.65$ (d, $J=$ $2.8 \mathrm{~Hz}, 1 \mathrm{H}), 7.61(\mathrm{dd}, J=6.8,1.8 \mathrm{~Hz}, 1 \mathrm{H}), 7.48(\mathrm{td}, J=9.2$, $6.8,2.4 \mathrm{~Hz}, 1 \mathrm{H}), 7.38(\mathrm{dd}, J=8.8,2.8 \mathrm{~Hz}, 1 \mathrm{H}), 7.21(\mathrm{~d}, J=$ $8.8 \mathrm{~Hz}, 1 \mathrm{H}), 6.45(\mathrm{~d}, J=9.2 \mathrm{~Hz}, 1 \mathrm{H}), 6.28(\mathrm{td}, J=6.8$, $1.2 \mathrm{~Hz}, 1 \mathrm{H}), 3.89(\mathrm{~s}, 3 \mathrm{H}) ;{ }^{13} \mathrm{C}$ NMR $\left(100 \mathrm{MHz}, \mathrm{CDCl}_{3}\right) \delta$ $162.1,155.5,139.7,137.6,133.9,131.1,126.5,121.5$, 111.6, 111.5, 105.8, 56.4; IR (KBr): 3100, 3062, 3024, 2988, 2939, 2363, 2337, $1661(\mathrm{C}=\mathrm{O}), 1585,1535$, $1485 \mathrm{~cm}^{-1}$; FAB (NBA) $280.0\left(\mathrm{M}^{+}+\mathrm{H}\right)$; HRMS calcd for $\mathrm{C}_{12} \mathrm{H}_{11}^{79} \mathrm{BrNO}_{2} 279.9973$, found $279.9971\left(\mathrm{M}^{+}+\mathrm{H}\right)$, calcd for $\mathrm{C}_{12} \mathrm{H}_{10}^{79} \mathrm{BrNO}_{2} 278.9895$, found $278.9896\left(\mathrm{M}^{+}\right)$. Anal. calcd for $\mathrm{C}_{12} \mathrm{H}_{10} \mathrm{BrNO}_{2} \mathrm{C}, 51.45 ; \mathrm{H}, 3.60 ; \mathrm{N}, 5.00$. Found C, 51.19; H, 3.58; N, 5.03.

2.3.10. 1-(4-(Diphenylamino)phenyl)pyrid-2-one (15). Reaction time: $24 \mathrm{~h}$, white solid (59\%). Mp $220-221^{\circ} \mathrm{C}$ (from $\mathrm{CH}_{2} \mathrm{Cl}_{2} /$ hexane $=1: 10$ ); ${ }^{1} \mathrm{H}$ NMR $\left(400 \mathrm{MHz}, \mathrm{d}_{6}\right.$ acetone) $\delta 7.56(\mathrm{ddd}, J=6.4,2.4,0.8 \mathrm{~Hz}, 1 \mathrm{H}), 7.44$ (ddd, $J=9.2,6.7,2.4 \mathrm{~Hz}, 1 \mathrm{H}), 7.29-7.36(\mathrm{~m}, 6 \mathrm{H}), 7.05-7.13(\mathrm{~m}$, $8 \mathrm{H}), 6.44(\mathrm{dd}, J=9.2,0.8 \mathrm{~Hz}, 1 \mathrm{H}), 6.26(\mathrm{td}, J=6.7,1.2 \mathrm{~Hz}$, $1 \mathrm{H}) ;{ }^{13} \mathrm{C}$ NMR $\left(100 \mathrm{MHz}, \mathrm{CDCl}_{3}\right) \delta 162.3,147.7,147.0$, 139.5, 138.0, 134.3, 129.2, 126.9, 124.8, 123.4, 122.7, 121.6, 105.9; $\mathrm{IR}(\mathrm{KBr}): 3076,3038,2363,2337,1661(\mathrm{C}=\mathrm{O}), 1585$, $1485,1473 \mathrm{~cm}^{-1}$; FAB (NBA) $339.1\left(\mathrm{M}^{+}+\mathrm{H}\right), 338.1\left(\mathrm{M}^{+}\right)$; HRMS calcd for $\mathrm{C}_{23} \mathrm{H}_{19} \mathrm{~N}_{2} \mathrm{O} 339.1497$, found 339.1493 $\left(\mathrm{M}^{+}+\mathrm{H}\right)$, calcd for $\mathrm{C}_{23} \mathrm{H}_{18} \mathrm{~N}_{2} \mathrm{O} 338.1419$, found 338.1417 $\left(\mathrm{M}^{+}\right)$. Anal. calcd for $\mathrm{C}_{23} \mathrm{H}_{18} \mathrm{~N}_{2} \mathrm{O}: \mathrm{C}, 81.63 ; \mathrm{H}, 5.36 ; \mathrm{N}, 8.28$. Found C, 81.48; H, 5.26; N, 8.41.

2.3.11. 1-(4'-(Diphenylamino)biphenyl-4-yl)-1 $H$-pyridin2-one (16). Reaction time: $20 \mathrm{~h}$, white solid (79\%). Mp 232-234 ${ }^{\circ} \mathrm{C}$ (from $\mathrm{CH}_{2} \mathrm{Cl}_{2} /$ hexane $=1: 10$ ); ${ }^{1} \mathrm{H}$ NMR (400 MHz, $\mathrm{d}_{6}$-DMSO) $\delta 7.74(\mathrm{dt}, J=8.8,2.2 \mathrm{~Hz}, 2 \mathrm{H})$, $7.68(\mathrm{dd}, J=7,1.4 \mathrm{~Hz}, 1 \mathrm{H}), 7.64(\mathrm{dt}, J=8.8,2.2 \mathrm{~Hz}, 2 \mathrm{H})$, $7.51(\mathrm{ddd}, J=8.8,6.7,2.2 \mathrm{~Hz}, 1 \mathrm{H}), 7.45(\mathrm{dt}, J=8.8,2.2 \mathrm{~Hz}$, 2H), 7.33 (t, $J=9.2 \mathrm{~Hz}, 4 \mathrm{H}), 7.02-7.09(\mathrm{~m}, 8 \mathrm{H}), 6.48(\mathrm{~d}$, $J=8.8 \mathrm{~Hz}, 1 \mathrm{H}), 6.32(\mathrm{td}, J=6.7,0.8 \mathrm{~Hz}, 1 \mathrm{H}), 6.32(\mathrm{td}, J=$ $6.7,0.8 \mathrm{~Hz}, 1 \mathrm{H}) ;{ }^{13} \mathrm{C}$ NMR $\left(100 \mathrm{MHz}, \mathrm{CDCl}_{3}\right) \delta 162.3$, $147.4,147.3,140.8,139.7,139.3,137.8,133.5,129.2$, 127.7, 127.3, 126.6, 124.5, 123.4, 123.0, 121.8, 105.9; IR (KBr): 3076, 3038, 2363, 2337, $1661(\mathrm{C}=\mathrm{O}), 1585,1485$, $1473 \mathrm{~cm}^{-1}$; FAB (NBA) $415.1\left(\mathrm{M}^{+}+\mathrm{H}\right)$; HRMS calcd for $\mathrm{C}_{29} \mathrm{H}_{22} \mathrm{~N}_{2} \mathrm{O}$ 414.1732, found $414.1735\left(\mathrm{M}^{+}\right)$. Anal. calcd for $\mathrm{C}_{29} \mathrm{H}_{22} \mathrm{~N}_{2} \mathrm{O}: \mathrm{C}, 84.03 ; \mathrm{H}, 5.35 ; \mathrm{N}, 6.76$. Found $\mathrm{C}, 83.74$; $\mathrm{H}, 5.22 ; \mathrm{N}, 7.08$.

2.3.12. 1-(Pyridin-3-yl)-1H-pyridinin-2-one (17). ${ }^{21}$ Reaction time: $15 \mathrm{~h}$, tint brown solid $(76 \%)$. Mp $144-145^{\circ} \mathrm{C}$ (from $\mathrm{CH}_{2} \mathrm{Cl}_{2}$ /hexane $\left.=1: 10\right) ;{ }^{1} \mathrm{H}$ NMR $\left(400 \mathrm{MHz}, \mathrm{d}_{6}-\right.$ DMSO) $\delta 8.61-8.63(\mathrm{~m}, 2 \mathrm{H}), 7.91(\mathrm{~d}, J=8.8 \mathrm{~Hz}, 1 \mathrm{H}), 7.71$ $(\mathrm{dt}, J=6.8,0.8 \mathrm{~Hz}, 1 \mathrm{H}), 7.50-7.57(\mathrm{~m}, 2 \mathrm{H}), 6.51(\mathrm{~d}, J=$ $8.8 \mathrm{~Hz}, 1 \mathrm{H}), 6.35(\mathrm{t}, J=6.8 \mathrm{~Hz}, 1 \mathrm{H}) ;{ }^{13} \mathrm{C} \mathrm{NMR}(100 \mathrm{MHz}$, $\mathrm{d}_{6}$-DMSO) $\delta 160.8,148.7,147.2,140.8,138.5,137.0$, 
134.4, 123.6, 120.3, 105.8; IR (KBr): 3063, 3012, 2363, 2337, $1661(\mathrm{C}=\mathrm{O}), 1573,1535 \mathrm{~cm}^{-1}$; FAB (NBA) 173.1 $\left(\mathrm{M}^{+}+\mathrm{H}\right)$; HRMS calcd for $\mathrm{C}_{10} \mathrm{H}_{9} \mathrm{~N}_{2} \mathrm{O}$ 173.0715, found 173.0712 $\left(\mathrm{M}^{+}+\mathrm{H}\right)$. Anal. calcd for $\mathrm{C}_{10} \mathrm{H}_{8} \mathrm{~N}_{2} \mathrm{O}$ : C, 69.76; H, 4.68; N, 16.27. Found C, 69.63; H, 4.60; N, 15.97.

2.3.13. 1-(6-(2-Oxopyridin-1(2H)-yl)pyridin-2-yl)pyridin-2(1H)-one (18). Reaction time: $4 \mathrm{~h}$, white solid (65\%). Mp 176-177 ${ }^{\circ} \mathrm{C}$ (from $\mathrm{CH}_{2} \mathrm{Cl}_{2} /$ hexane $=1: 10$ ); ${ }^{1} \mathrm{H}$ NMR (400 MHz, $\left.\mathrm{CDCl}_{3}\right) \delta 7.89-7.97(\mathrm{~m}, 3 \mathrm{H}), 7.79$ (dd, $J=$ $6.8,2 \mathrm{~Hz}, 2 \mathrm{H}), 7.36(\mathrm{ddd}, J=9.2,6.8,2 \mathrm{~Hz}, 2 \mathrm{H}), 6.60(\mathrm{~d}$, $J=9.2 \mathrm{~Hz}, 2 \mathrm{H}), 6.25(\mathrm{t}, J=6.8 \mathrm{~Hz}, 2 \mathrm{H}) ;{ }^{13} \mathrm{C} \mathrm{NMR}$ $\left(100 \mathrm{MHz}, \mathrm{CDCl}_{3}\right) \delta 161.7,150.6,140.1,139.3,135.6$, 121.9, 120.4, 106.3; IR (KBr): 3100, 3051, 2363, 2337, $1673(\mathrm{C}=\mathrm{O}), 1599,1573,1535 \mathrm{~cm}^{-1}$; FAB (NBA) 266.0 $\left(\mathrm{M}^{+}+\mathrm{H}\right)$; HRMS calcd for $\mathrm{C}_{15} \mathrm{H}_{12} \mathrm{~N}_{3} \mathrm{O}_{2}$ 266.0930, found 266.0922 $\left(\mathrm{M}^{+}+\mathrm{H}\right)$, calcd for $\mathrm{C}_{15} \mathrm{H}_{11} \mathrm{~N}_{3} \mathrm{O}_{2} 265.0851$, found 265.0848 $\left(\mathrm{M}^{+}\right)$. Anal. calcd for $\mathrm{C}_{15} \mathrm{H}_{11} \mathrm{~N}_{3} \mathrm{O}_{2}$ : C, 67.92; $\mathrm{H}$, 4.18; N, 15.84. Found C, 67.66; H, 4.09; N, 15.68.

2.3.14. 1-(Thiazol-2-yl)-1H-pyridin-2-one (19). Reaction time: $14 \mathrm{~h}$, tint yellow solid (33\%). Mp 85.5-86 ${ }^{\circ} \mathrm{C}$ (from $\mathrm{CH}_{2} \mathrm{Cl}_{2}$ /hexane $\left.=1: 15\right) ;{ }^{1} \mathrm{H}$ NMR (400 MHz, $\mathrm{d}_{6}$-DMSO) $\delta$ 8.76 (ddd, $J=7.6,2,0.8 \mathrm{~Hz}, 1 \mathrm{H}), 7.77$ (d, $J=3.2 \mathrm{~Hz}, 1 \mathrm{H})$, $7.59-7.64(\mathrm{~m}, 2 \mathrm{H}), 6.73(\mathrm{dd}, J=8,0.8 \mathrm{~Hz}, 1 \mathrm{H}), 6.58(\mathrm{td}$, $J=7.6,0.8 \mathrm{~Hz}, 1 \mathrm{H}) ;{ }^{13} \mathrm{C} \mathrm{NMR}\left(100 \mathrm{MHz}, \mathrm{CDCl}_{3}\right) \delta 160.1$, $155.7,139.3,137.5,131.6,121.2,118.4,107.2$; IR (KBr): 3114, 3068, 2357, 2335, $1670(\mathrm{C}=\mathrm{O}), 1599,1541$, $1495 \mathrm{~cm}^{-1}$; FAB (NBA) $179.0\left(\mathrm{M}^{+}+\mathrm{H}\right)$; HRMS calcd for $\mathrm{C}_{8} \mathrm{H}_{7} \mathrm{~N}_{2} \mathrm{OS}$ 179.0279, found $179.0282\left(\mathrm{M}^{+}+\mathrm{H}\right)$, calcd for $\mathrm{C}_{8} \mathrm{H}_{6} \mathrm{~N}_{2} \mathrm{OS}$ 178.0201, found $178.0204\left(\mathrm{M}^{+}\right)$. Anal. calcd for $\mathrm{C}_{8} \mathrm{H}_{6} \mathrm{~N}_{2} \mathrm{OS}$ : C, 53.92; H, 3.39; N, 15.72; S, 17.99 . Found C, 53.97; H, 3.40; N, 15.59; S, 17.67.

2.3.15. 1-(Thiophen-3-yl)-1H-pyridin-2-one (20). ${ }^{17}$ Reaction time: $22 \mathrm{~h}$, brown solid (74\%). Mp 116-117 ${ }^{\circ} \mathrm{C}$ (from $\mathrm{CH}_{2} \mathrm{Cl}_{2}$ /hexane $\left.=1: 15\right) ;{ }^{1} \mathrm{H}$ NMR (400 MHz, $\mathrm{d}_{6}$-DMSO) $\delta$ $7.75(\mathrm{dd}, J=3.2,1.4 \mathrm{~Hz}, 1 \mathrm{H}), 7.70(\mathrm{dd}, J=6.8,2.4 \mathrm{~Hz}, 1 \mathrm{H})$, $7.62(\mathrm{dd}, J=5.2,3.2 \mathrm{~Hz}, 1 \mathrm{H}), 7.47$ (ddd, $J=9.2,6.8$, $2.4 \mathrm{~Hz}, 1 \mathrm{H}), 7.28(\mathrm{dd}, J=5,1.4 \mathrm{~Hz}, 1 \mathrm{H}), 6.47(\mathrm{~d}, J=$ $9.2 \mathrm{~Hz}, 1 \mathrm{H}), 6.29(\mathrm{td}, J=6.8,1.4 \mathrm{~Hz}, 1 \mathrm{H}) ;{ }^{13} \mathrm{C} \mathrm{NMR}$ $\left(100 \mathrm{MHz}, \mathrm{CDCl}_{3}\right) \delta 161.8,139.4,138.6,137.4,125.4$, 124.8, 121.8, 119.6, 106.0; IR (KBr): 3112, 3066, 2361, 2333, $1661(\mathrm{C}=\mathrm{O}), 1583,1515 \mathrm{~cm}^{-1}$; FAB (NBA) 178.0 $\left(\mathrm{M}^{+}+\mathrm{H}\right)$; HRMS calcd for $\mathrm{C}_{9} \mathrm{H}_{8} \mathrm{NOS} 178.0327$, found 178.0326 $\left(\mathrm{M}^{+}+\mathrm{H}\right)$. Anal. calcd for $\mathrm{C}_{9} \mathrm{H}_{7} \mathrm{NOS}$ : C, 60.99; $\mathrm{H}$, 3.98; N, 7.90; S, 18.09. Found C, 61.00; H, 3.95; N, 7.83; S, 18.15 .

2.3.16. 1-(3-Nitrophenyl)-1H-pyridin-2-one (21). ${ }^{22}$ Reaction time: $15 \mathrm{~h}$, tint yellow solid $(62 \%)$. Mp $185-186{ }^{\circ} \mathrm{C}$ (from $\mathrm{CH}_{2} \mathrm{Cl}_{2} /$ hexane $\left.=1: 10\right)$; ${ }^{1} \mathrm{H} \mathrm{NMR}$ (400 MHz, $\mathrm{CDCl}_{3}$ ) $\delta 8.27-8.27(\mathrm{~m}, 2 \mathrm{H}), 7.79(\mathrm{dt}, J=8,1.6 \mathrm{~Hz}, 1 \mathrm{H}), 7.67$ $(\mathrm{t}, J=8 \mathrm{~Hz}, 1 \mathrm{H}), 7.43$ (ddd, $J=9.2,6.6,2.4 \mathrm{~Hz}, 1 \mathrm{H}), 7.33$ $(\mathrm{dd}, J=6.6,1.6 \mathrm{~Hz}, 1 \mathrm{H}), 6.67(\mathrm{~d}, J=9.2 \mathrm{~Hz}, 1 \mathrm{H}), 6.30(\mathrm{t}$, $J=6.6 \mathrm{~Hz}, 1 \mathrm{H}) ;{ }^{13} \mathrm{C} \mathrm{NMR}\left(100 \mathrm{MHz}, \mathrm{CDCl}_{3}\right) \delta 161.7$, $148.4,141.4,140.3,136.7,132.8,130.0,123.2,122.1$, 121.9, 106.7; IR (KBr): 3080, 3023, 2361, 2333, 1671 $(\mathrm{C}=\mathrm{O}), 1593,1525 \mathrm{~cm}^{-1}$; FAB (NBA) $217.0\left(\mathrm{M}^{+}+\mathrm{H}\right)$; HRMS calcd for $\mathrm{C}_{11} \mathrm{H}_{9} \mathrm{~N}_{2} \mathrm{O}_{3} 217.0613$, found 217.0612 $\left(\mathrm{M}^{+}+\mathrm{H}\right)$, calcd for $\mathrm{C}_{11} \mathrm{H}_{8} \mathrm{~N}_{2} \mathrm{O}_{3} 216.0535$, found 216.0534 $\left(\mathrm{M}^{+}\right)$. Anal. calcd for $\mathrm{C}_{11} \mathrm{H}_{8} \mathrm{~N}_{2} \mathrm{O}_{3}: \mathrm{C}, 61.11 ; \mathrm{H}, 3.73 ; \mathrm{N}$, 12.96. Found C, 61.30; H, 3.79; N, 12.88.
2.3.17. 1-(4-(Methylthio)phenyl)-1H-pyridin-2-one (22). Reaction time: $5 \mathrm{~h}$, white solid $(80 \%)$. Mp $141-142{ }^{\circ} \mathrm{C}$ (from $\mathrm{CH}_{2} \mathrm{Cl}_{2} /$ hexane $\left.=1: 10\right) ;{ }^{1} \mathrm{H} \mathrm{NMR}$ (400 MHz, $\mathrm{d}_{6^{-}}$ DMSO) $\delta 7.60(\mathrm{dd}, J=6.6,2 \mathrm{~Hz}, 1 \mathrm{H}), 7.48$ (ddd, $J=9.2$, 6.6, $2 \mathrm{~Hz}, 1 \mathrm{H}), 7.30-7.38(\mathrm{~m}, 4 \mathrm{H}), 6.45(\mathrm{~d}, J=9.2 \mathrm{~Hz}, 1 \mathrm{H})$, $6.29(\mathrm{td}, J=6.6,1.2 \mathrm{~Hz}, 1 \mathrm{H}), 2.5(\mathrm{~s}, 3 \mathrm{H}) ;{ }^{13} \mathrm{C} \mathrm{NMR}$ $\left(100 \mathrm{MHz}, \mathrm{CDCl}_{3}\right) \delta 162.0,139.6,139.1,137.6,137.4$, 126.6, 126.5, 121.5, 105.8, 15.7; IR (KBr): 3046, 2980, 2922, 2361, 2342, $1660(\mathrm{C}=\mathrm{O}), 1591,1525,1487 \mathrm{~cm}^{-1}$; FAB (NBA) $218.0\left(\mathrm{M}^{+}+\mathrm{H}\right)$; HRMS calcd for $\mathrm{C}_{12} \mathrm{H}_{12} \mathrm{NOS}$ 218.0640, found $218.0642\left(\mathrm{M}^{+}+\mathrm{H}\right)$, calcd for $\mathrm{C}_{12} \mathrm{H}_{11} \mathrm{NOS}$ 217.0561, found 217.0554 $\left(\mathrm{M}^{+}\right)$. Anal. calcd for $\mathrm{C}_{12} \mathrm{H}_{11} \mathrm{NOS}$ : C, 66.33; H, 5.10; N, 6.45; S, 14.76. Found C, 66.36; H, 4.96; N, 6.40; S, 14.91 .

2.3.18. 5-Nitro-1-phenyl-1H-pyridin-2-one. Reaction time: $16 \mathrm{~h}$, tint yellow solid (3\%). Mp $169.5-170{ }^{\circ} \mathrm{C}$ (from $\mathrm{CH}_{2} \mathrm{Cl}_{2}$ /hexane $\left.=1: 10\right) ;{ }^{1} \mathrm{H}$ NMR $\left(400 \mathrm{MHz} \mathrm{CDCl}_{3}\right) \delta 8.64$ $(\mathrm{d}, J=3.2 \mathrm{~Hz}, 1 \mathrm{H}), 8.14(\mathrm{dd}, J=10.4,3.2 \mathrm{~Hz}, 1 \mathrm{H}), 7.47-$ $7.56(\mathrm{~m}, 3 \mathrm{H}), 7.36-7.39(\mathrm{~m}, 2 \mathrm{H}), 6.64(\mathrm{~d}, J=10.4 \mathrm{~Hz}, 1 \mathrm{H})$; ${ }^{13} \mathrm{C} \mathrm{NMR}\left(100 \mathrm{MHz}, \mathrm{CDCl}_{3}\right) \delta 160.9,139.6,139.0,133.3$, 130.8, 129.64, 129.61, 126.1, 120.3; IR (KBr): 3085, 2361, 2333, $1667(\mathrm{C}=\mathrm{O}), 1621,1553,1497,1450 \mathrm{~cm}^{-1}$; FAB (NBA) $217.0\left(\mathrm{M}^{+}+\mathrm{H}\right)$; HRMS calcd for $\mathrm{C}_{11} \mathrm{H}_{9} \mathrm{~N}_{2} \mathrm{O}_{3}$ 217.0613, found $217.0613\left(\mathrm{M}^{+}+\mathrm{H}\right)$, calcd for $\mathrm{C}_{11} \mathrm{H}_{8} \mathrm{~N}_{2} \mathrm{O}_{3} 216.0535$, found $216.0530\left(\mathrm{M}^{+}\right)$. Anal. calcd for $\mathrm{C}_{11} \mathrm{H}_{8} \mathrm{~N}_{2} \mathrm{O}_{3} \mathrm{C}, 61.11 ; \mathrm{H}, 3.73 ; \mathrm{N}, 12.96$. Found C, 60.91; $\mathrm{H}, 3.76$; N, 12.62 .

2.3.19. 2-tert-Butoxy-5-bromopyridine (26). To an ovendried $100 \mathrm{~mL}$ double-necked flask was charged a solution of 2,5-dibromopyridine $(4.90 \mathrm{~g}, 21 \mathrm{mmol})$ and sodium tertbutoxide $(3.25 \mathrm{~g}, 30 \mathrm{mmol})$ in toluene $(30 \mathrm{ml})$. The reaction mixture was stirred and heated at reflux temperature for $2.5 \mathrm{~h}$. The resulting suspension was cooled to room temperature and filtered through celite. The collected organic phase was concentrated, and purified by flash chromatography on silica gel (hexane/dichloromethane) to give colorless liquid $(2.65 \mathrm{~g}, 56 \%)$. Note that the compound is a slightly hygroscopic liquid and should be distilled before use. Bp $70{ }^{\circ} \mathrm{C}(0.15 \mathrm{mmHg}) .{ }^{1} \mathrm{H} \mathrm{NMR}\left(400 \mathrm{MHz}, \mathrm{d}_{6^{-}}\right.$ DMSO) $\delta 8.20(\mathrm{~d}, J=2.4 \mathrm{~Hz}, 1 \mathrm{H}), 7.80(\mathrm{dd}, J=8.8,2.8 \mathrm{~Hz}$, $1 \mathrm{H}), 6.66(\mathrm{~d}, J=8.8 \mathrm{~Hz}, 1 \mathrm{H}), 1.50(\mathrm{~s}, 9 \mathrm{H}) ;{ }^{13} \mathrm{C} \mathrm{NMR}$ $\left(100 \mathrm{MHz}, \mathrm{CDCl}_{3}\right) \delta 162.4,146.8,140.5,114.9,111.2$, 80.2, 28.6; IR (neat): 2980, 2933, 2353, 2343, 1573, 1545, $1459,1363 \mathrm{~cm}^{-1}$; HRMS (EI, $70 \mathrm{eV}$ ) calcd for $\mathrm{C}_{9} \mathrm{H}_{12}^{79} \mathrm{BrNO}$ 229.0102, found $229.0097\left(\mathrm{M}^{+}\right)$. Anal. calcd for $\mathrm{C}_{9} \mathrm{H}_{12^{-}}$ BrNOC, 46.98; H, 5.26; N, 6.09. Found C, 46.93; H, 5.41; N, 5.93 .

2.3.20. 1-(6-tert-Butoxypyridin-3-yl)-1H-pyridin-2-one (27). To an oven-dried $25 \mathrm{~mL}$ double-necked flask was charged 2-hydroxypyridine (1.26 g, $13.2 \mathrm{mmol}), \mathrm{CuI}$ (20 mol\%), and $\mathrm{K}_{2} \mathrm{CO}_{3}$ (2 equiv). The flask was evacuated and backfilled with nitrogen. A solution of 2-tert-butoxy-5bromopyridine $(3.03 \mathrm{~g}, 13.2 \mathrm{mmol})$ in toluene $(13.2 \mathrm{~mL})$ and DMCDA (20 mol\%) were injected under nitrogen. The reaction mixture was stirred and heated at reflux temperature for $11 \mathrm{~h}$. The resulting mixture was cooled to room temperature, diluted with dichloromethane, and filtered. The filtrate was washed with water. The collected organic phase was dried over anhydrous $\mathrm{MgSO}_{4}$ and concentrated. The crude product was purified by flash chromatography on 
silica gel (ethyl acetate/dichloromethane) to give white solid (2.03 g, 63\%). Mp $188-189{ }^{\circ} \mathrm{C}$ (from $\mathrm{CH}_{2} \mathrm{Cl}_{2} /$ hexane $=$ $1: 10) ;{ }^{1} \mathrm{H}$ NMR (400 MHz, $\mathrm{d}_{6}$-acetone) $\delta 8.14(\mathrm{~d}, J=$ $2.8 \mathrm{~Hz}, 1 \mathrm{H}), 7.71$ (dd, $J=9.2,2.8 \mathrm{~Hz}, 1 \mathrm{H}), 7.57$ (ddd, $J=$ $6.8,2,0.8 \mathrm{~Hz}, 1 \mathrm{H}), 7.47$ (ddd, $J=9.2,6.8,2 \mathrm{~Hz}, 1 \mathrm{H}), 6.75$ $(\mathrm{d}, J=9.2 \mathrm{~Hz}, 1 \mathrm{H}), 6.45(\mathrm{dt}, J=9.2,0.8 \mathrm{~Hz}, 1 \mathrm{H}), 6.28(\mathrm{td}$, $J=6.8,1.2 \mathrm{~Hz}, 1 \mathrm{H}), 1.60(\mathrm{~s}, 9 \mathrm{H}) ;{ }^{13} \mathrm{C} \mathrm{NMR}(100 \mathrm{MHz}$, $\left.\mathrm{CDCl}_{3}\right) \delta 163.1,162.2,143.1,139.9,137.8,136.5,130.6$, 121.5, 113.1, 106.0, 80.3, 28.6; IR (KBr): 3047, 2971, 2923, 2361, 2333, $1667(\mathrm{C}=\mathrm{O}), 1583,1535,1477 \mathrm{~cm}^{-1}$; FAB (NBA) $245.1\left(\mathrm{M}^{+}+\mathrm{H}\right)$; HRMS calcd for $\mathrm{C}_{14} \mathrm{H}_{17} \mathrm{~N}_{2} \mathrm{O}_{2}$ 245.1290, found 245.1289 $\left(\mathrm{M}^{+}+\mathrm{H}\right)$. Anal. calcd for $\mathrm{C}_{14} \mathrm{H}_{16} \mathrm{~N}_{2} \mathrm{O}_{2}$ : $\mathrm{C}, 68.83 ; \mathrm{H}, 6.60 ; \mathrm{N}$, 11.47. Found $\mathrm{C}$, 68.77; H, 6.82; N, 11.40 .

2.3.21. 1-(6-Hydroxypyridin-3-yl)-1H-pyridin-2-one (28). To an oven-dried $25 \mathrm{~mL}$ flask was charged 1-(6-tertbutoxypyridin-3-yl)pyridin-2(1H)-one $(0.75 \mathrm{~g}, 3.06 \mathrm{mmol})$, $\mathrm{CH}_{2} \mathrm{Cl}_{2}(15 \mathrm{~mL})$, and TFA $(5 \%)$. The reaction mixture was stirred and heated at reflux temperature for $1 \mathrm{~h}$. The resulting solution was concentrated and washed with hexane to give white solid. Note that this compound is hygroscopic and soluble in water. Normal extraction workup procedure is inappropriate in this case. The white solid was dried under vacuum at $110^{\circ} \mathrm{C}$ for overnight to afford $0.57 \mathrm{~g}, 99 \%$ yield of 28. Mp 284-285 ${ }^{\circ} \mathrm{C}$ (from $\mathrm{CH}_{2} \mathrm{Cl}_{2} /$ hexane $=1: 5$ ); ${ }^{1} \mathrm{H}$ NMR (400 MHz, d 6 -DMSO) $\delta 12.07$ (bs, 1H), 7.60-7.63 $(\mathrm{m}, 2 \mathrm{H}), 7.44-7.51(\mathrm{~m}, 2 \mathrm{H}), 6.44(\mathrm{~d}, J=9.6 \mathrm{~Hz}, 1 \mathrm{H}), 6.40$ $(\mathrm{d}, J=10 \mathrm{~Hz}, 1 \mathrm{H}), 6.26(\mathrm{td}, J=6.8,1.2 \mathrm{~Hz}, 1 \mathrm{H}) ;{ }^{13} \mathrm{C} \mathrm{NMR}$ (100 MHz, d $\mathrm{d}_{6}$-DMSO) $\delta 161.3,161.2,140.6,140.5,139.4$, 133.7, 121.7, 121.0, 118.6, 105.5; IR (KBr): 3446, 3140, 3065, 2799, 2353, $1677(\mathrm{C}=\mathrm{O}), 1621,1583,1525$, $1469 \mathrm{~cm}^{-1}$; FAB (NBA) $189.1\left(\mathrm{M}^{+}+\mathrm{H}\right)$; HRMS calcd for $\mathrm{C}_{10} \mathrm{H}_{9} \mathrm{~N}_{2} \mathrm{O}_{2} 189.0664$, found $189.0667\left(\mathrm{M}^{+}+\mathrm{H}\right)$, calcd for $\mathrm{C}_{10} \mathrm{H}_{8} \mathrm{~N}_{2} \mathrm{O}_{2}$ 188.0586, found $188.0587\left(\mathrm{M}^{+}\right)$. Anal. calcd for $\mathrm{C}_{10} \mathrm{H}_{8} \mathrm{~N}_{2} \mathrm{O}_{2}$ : C, 63.82; H, 4.28; N, 14.89. Found $\mathrm{C}$, $63.41 ; \mathrm{H}, 4.57$; N, 15.01 .

2.3.22. 1-(1-(6-tert-Butoxypyridin-3-yl)-1,6-dihydro-6oxopyridin-3-yl)-1H-pyridin-2-one (29). To an ovendried $10 \mathrm{~mL}$ double-necked flask containing a stir-bar was charged with 1-(6-hydroxypyridin-3-yl)-1H-pyridin-2-one $(0.19 \mathrm{~g}, 1.01 \mathrm{mmol}), \mathrm{CuI}(20 \mathrm{~mol} \%)$, and $\mathrm{K}_{2} \mathrm{CO}_{3}$ (2 equiv). The flask was evacuated and backfilled with nitrogen. A solution of 2-tert-butoxy-5-bromopyridine (0.28 g, $1.21 \mathrm{mmol})$ in toluene $(1 \mathrm{~mL})$ and DMCDA $(20 \mathrm{~mol} \%)$ were injected under nitrogen. The reaction mixture was stirred and heated at reflux temperature for $12 \mathrm{~h}$. The resulting mixture was cooled to room temperature, diluted with dichloromethane, and filtered. The filtrate was washed with water. The collected organic phase was dried over anhydrous $\mathrm{MgSO}_{4}$ and concentrated. The crude product was purified by flash chromatography on silica gel (ethyl acetate/ dichloromethane/MeOH) to give white solid $(0.28 \mathrm{~g}, 82 \%)$. Mp 216-217 ${ }^{\circ} \mathrm{C}$ (from $\mathrm{CH}_{2} \mathrm{Cl}_{2} /$ hexane $=1: 10$ ); ${ }^{1} \mathrm{H}$ NMR (400 MHz, d ${ }_{6}$ DMSO) $\delta 8.22(\mathrm{~d}, J=2.8 \mathrm{~Hz}, 1 \mathrm{H}), 8.00(\mathrm{~d}$, $J=2.8 \mathrm{~Hz}, 1 \mathrm{H}), 7.78(\mathrm{dd}, J=8.8,2.8 \mathrm{~Hz}, 1 \mathrm{H}), 7.73(\mathrm{dd}, J=$ $7.2,2 \mathrm{~Hz}, 1 \mathrm{H}), 7.62(\mathrm{dd}, J=10,2.8 \mathrm{~Hz}, 1 \mathrm{H}), 7.49$ (ddd, $J=$ $9.2,6.8,2 \mathrm{~Hz}, 1 \mathrm{H}), 6.80(\mathrm{~d}, J=8.8 \mathrm{~Hz}, 1 \mathrm{H}), 6.53(\mathrm{~d}, J=$ $10 \mathrm{~Hz}, 1 \mathrm{H}), 6.46(\mathrm{~d}, J=9.2 \mathrm{~Hz}, 1 \mathrm{H}), 6.29$ (td, $J=6.8$, $1.2 \mathrm{~Hz}, 1 \mathrm{H}), 1.60(\mathrm{~s}, 9 \mathrm{H}) ;{ }^{13} \mathrm{C} \mathrm{NMR}\left(100 \mathrm{MHz}, \mathrm{CDCl}_{3}\right) \delta$ $163.4,162.1,161.0,143.2,140.3,139.1,137.3,136.4$, $135.5,130.0,122.0,121.7,121.5,113.3,106.5,80.6,28.6$;
IR (KBr): 3055, 2970, 2361, 2333, $1679(\mathrm{C}=\mathrm{O}), 1661$ $(\mathrm{C}=\mathrm{O}), 1619,1573,1525,1463 \mathrm{~cm}^{-1}$; FAB (NBA) 338.2 $\left(\mathrm{M}^{+}+\mathrm{H}\right)$; HRMS calcd for $\mathrm{C}_{19} \mathrm{H}_{20} \mathrm{~N}_{3} \mathrm{O}_{3} 338.1505$, found $338.1503\left(\mathrm{M}^{+}+\mathrm{H}\right)$, calcd for $\mathrm{C}_{19} \mathrm{H}_{19} \mathrm{~N}_{3} \mathrm{O}_{3} 337.1426$, found $337.1439\left(\mathrm{M}^{+}\right)$. Anal. calcd for $\mathrm{C}_{19} \mathrm{H}_{19} \mathrm{~N}_{3} \mathrm{O}_{3} \mathrm{C}, 67.64 ; \mathrm{H}$, 5.68; N, 12.45. Found C, 67.40; H, 5.63; N, 12.59.

\section{Acknowledgements}

We thank the National Science Council of Republic of China for the financial support (NSC 93-2113-M-002-008).

\section{Supplementary data}

Supplementary data associated with this article can be found, in the online version, at doi:10.1016/j.tet.2005. 01.063

\section{References and notes}

1. For reviews, see (a) Sieburth, S. McN. In The Inter- and Intramolecular [4+4] Photocycloaddition of 2-Pyridones and Its Application to Natural Product Synthesis; Harmata, M., Ed.; Advances in Cycloaddition; JAI: Greenwich, CT, 1999; Vol. 5, p 85. (b) Mehta, G.; Singh, V. Chem. Rev. 1999, 99, 881-930. (c) Joule, J. A.; Mills, K.; Smith, G. F. Heterocyclic Chemistry, 3rd ed.; Chapman and Hall: London, 1995; pp 87-93. (d) Kuzuya, M.; Noguchi, A. Trends Org. Chem. 1991, 2, 73 .

2. (a) Kohmoto, S.; Noguchi, T.; Masu, H.; Kishikawa, K.; Yamamoto, M.; Yamaguchi, K. Org. Lett. 2004, 6, 683-685. (b) Tanaka, K.; Fujiwara, T.; Urbanczyk-Lipkowska, Z. Org. Lett. 2002, 4, 3255-3257. (c) Zhu, M.; Qiu, Z.; Hiel, G. P.; Sieburth, S.; McN J. Org. Chem. 2002, 67, 3487-3493. (d) Ader, T. A.; Champey, C. A.; Kuznetsova, L. V.; Li, T.; Lim, Y.-H.; Rucando, D.; Sieburth, S. McN. Org. Lett. 2001, 3, 2165-2167. (e) Lee, Y.-g.; McGee, K. F., Jr.; Chen, J.; Rucando, D.; Sieburth, S. McN. J. Org. Chem. 2000, 65, 6676-6681. (f) Sieburth, S. McN. ; McGee, K. F., Jr.; Zhang, F.; Chen, Y. J. Org. Chem. 2000, 65, 1972-1977. (g) Bach, T.; Bergmann, H.; Harms, K. Org. Lett. 2001, 3, 601-603. (h) Bossharth, E.; Desbordes, P.; Monteiro, N.; Balme, G. Org. Lett. 2003, 5, 2441-2444.Margolin, S. B. US patents: 5,518,729 1996, 5,716,632, 1998; 5,962,478 1999, 6,090,822 2000; 6,114,353 2000.

3. (a) Itami, K.; Yamazaki, D.; Yoshida, J.-i. Org. Lett. 2003, 5, 2161-2164. (b) Yamamoto, Y.; Takagishi, H.; Itoh, K. Org. Lett. 2001, 3, 2117-2119. (c) O'Neill, B. T.; Yohannes, D.; Bundesmann, M. W.; Arnold, E. P. Org. Lett. 2000, 2, 4201-4204. (d) Moreno-Mañas, M.; Pleixats, R.; Villarroya, M. Tetrahedron 1993, 49, 1457-1464. (e) Katritzky, A. R.; Sengupta, S. Tetrahedron Lett. 1987, 28, 5419-5422. (f) Sugahara, M.; Moritani, Y.; Kuroda, T.; Kondo, K.; Shimadzu, H.; Ukita, T. Chem. Pharm. Bull. 2000, 48, 589-591.

4. (a) Sugahara, M.; Ukita, T. Chem. Pharm. Bull. 1997, 45, 
719-721. (b) Li, C. S.; Dixon, D. D. Tetrahedron Lett. 2004, 45, 4257-4260. (c) Renger, B. Synthesis 1985, 856-860. (d) Lam, P. Y. S.; Vincent, G.; Bonne, D.; Clark, C. G. Tetrahedron Lett. 2002, 43, 3091-3094.

5. Early copper catalyzed $\mathrm{C}-\mathrm{N}$ bond coupling was reported by Gilman, H.; Moore, L. O. J. Am. Chem. Soc. 1957, 79, 3485-3487.

6. (a) Wolfe, J. P.; Wagaw, S.; Marcoux, J.-F.; Buchwald, S. L. Acc. Chem. Res. 1998, 31, 805-818. (b) Hartwig, J. F. Angew. Chem., Int. Ed. 1998, 37, 2046-2067.

7. (a) Kwong, F. Y.; Buchwald, S. L. Org. Lett. 2003, 5, 793-796. (b) Kwong, F. Y.; Klapars, A.; Buchwald, S. L. Org. Lett. 2002, 4, 581-584.

8. (a) Gujadhur, R.; Venkataraman, D.; Kintigh, J. T. Tetrahedron Lett. 2001, 42, 4791-4793. (b) Gujadhur, R. K.; Bates, C. G.; Venkataraman, D. Org. Lett. 2001, 3, 4315-4317. (c) Goodbrand, H. B.; Hu, N.-X. J. Org. Chem. 1999, 64, 670-674. (d) Kelkar, A. A.; Patil, N. M.; Chaudhari, R. V. Tetrahedron Lett. 2002, 43, 7143-7146. (e) Lang, F.; Zewge, D.; Houpis, I. N.; Volante, R. P. Tetrahedron Lett. 2001, 42, 3251-3254.

9. (a) Klapars, A.; Antilla, J. C.; Huang, X.; Buchwald, S. L. J. Am. Chem. Soc. 2001, 123, 7727-7729. (b) Klapars, A.; Huang, X.; Buchwald, S. L. J. Am. Chem. Soc. 2002, 124, 7421-7428. (c) Antilla, J. C.; Klapars, A.; Buchwald, S. L. J. Am. Chem. Soc. 2002, 124, 11684-11688. (d) Kang, S.-K.; Kim, D.-H.; Park, J.-N. Synlett 2002, 427-430.

10. Kiyomori, A.; Marcoux, J.-F.; Buchwald, S. L. Tetrahedron Lett. 1999, 40, 2657-2660.
11. Wolter, M.; Klapars, A.; Buchwald, S. L. Org. Lett. 2001, 3, 3803-3805.

12. While DMF is a teratogen, dioxane is a carcinogen suspect agent.

13. (a) Leung, M.-k.; Mandal, A. B.; Wang, C.-C.; Lee, G.-H.; Peng, S.-M.; Cheng, H.-L.; Her, G.-R.; Chao, I.; Lu, H.-F.; Sun, Y.-C.; Shiao, M.-Y.; Chou, P.-T. J. Am. Chem. Soc. 2002, 124, 4287-4297. (b) Chien, C.-H.; Leung, M.-k.; Su, J.-K.; Li, G.-H.; Liu, Y.-H.; Wang, Y. J. Org. Chem. 2004, 69, 1866-1871.

14. Substitution of the bromo group by sterically less hindered alkoxides has been previous reported by several teams. For reference, see Tomioka, K.; Shinmi, Y.; Shiina, K.; Nakajima, M.; Koga, K. Chem. Pharm. Bull. 1990, 38, 2133-2135.

15. Taylor, E. C.; Harrison, K. A.; Rampal, J. B. J. Org. Chem. 1986, 51, 101-102.

16. Bain, B. M.; Saxton, J. E. J. Chem. Soc. 1961, 5216-5223.

17. Sugahara, M.; Ukita, T. Chem. Pharm. Bull. 1997, 45, 719-721.

18. Werner, D. D. M.; Christos, T.; Bertram, C.; Johannes, G.; Christopher, B. Eur. Pattern 2002, 57, 236.

19. Masakatsu, M.; Nobuko, W. JP Pattern 2003, 81, 977.

20. Jane, D. S.; Hutchinson, J. H.; Shaw, A. W.; Graham, S. L.; Ciccarone, T. M. Eur. Pattern 1999, 17, 777.

21. Kane, V. V.; Harvey, W.; Levine, S. D. J. Heterocyclic Chem. 1976, 13, 673-674.

22. Alicja, R.; Alicja, S.; Halina, B.-D. Acta Polon. Pharm. 1963, 20, 155-167. 